\title{
MedienPädagogik
}

Zeitschrift für Theorie und Praxis der Medienbildung www.medienpaed.com

ISSN 1424-3636

Themenheft Nr. 36: Teilhabe in einer durch digitale Medien geprägten Welt Perspektiven des wissenschaftlichen Nachwuchses

Herausgegeben von Tim Riplinger, Jan Hellriegel und Ricarda Bolten

\section{Informatik-Kompetenzen für technikferne Erwachsene zur Teilhabe in der Digitalen Welt}

Svenja Noichl

\begin{abstract}
Zusammenfassung
Um aktiv an der durch digitale Medien geprägten Welt teilhaben zu können, werden grundlegende Informatik-Kompetenzen immer wichtiger. Aufgrund der rasanten Entwicklung immer neuer Technologien, reichen reine Anwendungskenntnisse nicht mehr aus. Es ist genauso wichtig zu wissen, was dahinter steckt und die grundlegenden Konzepte hinter der Funktionalität zu kennen, um eine Übertragbarkeit von den heutigen Geräten, auf die Geräte von morgen zu ermöglichen. Nationale und internationale Schulcurricula und Standards geben vor, was Schülerinnen und Schüler in der Schule über Informatik und Informatiksysteme lernen sollen. Aussen vor stehen dabei jedoch diejenigen Menschen, die während ihrer Schulzeit, im Studium oder der beruflichen Aus- und Weiterbildung, keinen Kontakt zu irgendeiner Form von Informatikunterricht hatten. Ziel dieses Beitrags ist es daher basierend auf den vorhandenen Richtlinien für Schulen, Themen von aktuellem Interesse in Deutschland und dem Interesse der Zielgruppe, welches durch eine Befragung von 123 Personen ermittelt wurde, diejenigen Kompetenzen zu ermittelt, die technikferne Erwachsene erwerben sollten. Zum Erwerb dieser Kompetenzen wären beispielsweise Kurs- und Workshop-Angebote an Volkshochschulen denkbar.
\end{abstract}

Computer Science Competencies for Non-Technical Adults to Participate in the Digital World

\begin{abstract}
In order to actively participate in the world shaped by digital media, basic computer science skills are becoming increasingly important. Due to the rapid development of new technologies, pure application knowledge is no longer sufficient. It is just as important to know what lies behind it and to know the basic concepts behind the functionality in order to enable transferability from today's devices to tomorrow's devices. National and international school curricula and standards specify what students should learn about computer science and computing systems at school. However, those people who have had no contact with any form of computer science education during their time at school, during their studies or during vocational training and further education are out in front. The aim of this article is therefore to identify those competences, which should be acquired by non-
\end{abstract}

Noichl, Svenja. 2019. «Informatik-Kompetenzen für technikferne Erwachsene zur Teilhabe in der Digitalen Welt». MedienPädagogik 36, (November), 117-132. https://doi.org/10.21240/mpaed/36/2019.11.17.X. 
technical adults based on the existing guidelines for schools, topics of current interest in Germany and the interest of the target group, which was determined by a survey of 123 persons. To acquire these competences, courses and workshops may be offered at adult education centres for example.

\section{Einleitung und Motivation}

Digitalisierung hält in der heutigen Zeit verstärkt Einzug in alle Bereiche des täglichen Lebens. Das stellt gerade diejenigen Personen, die nur wenig Erfahrung im Umgang mit Technologien und digitalen Medien haben vor immer neue Herausforderungen. Ein grundlegendes Verständnis von informatischen Konzepten, das mehr umfasst als eine Bedienfähigkeit der gerade aktuellen digitalen Medien, könnte hier Problemen entgegenwirken. Mit einem grundlegenden Verständnis davon warum die Dinge, z.B. im Zusammenhang mit Smartphones oder Tablets, so funktionieren, wie sie funktionieren, ermöglicht eine bessere Übertragbarkeit und ein besseres Verständnis der digitalen Medien, denen wir in z.B. 10 Jahren gegenüberstehen werden. Denn die Technologie an sich mag sich weiterentwickeln, wir stehen aber sehr wahrscheinlich weiterhin ähnlichen Problematiken wie Fragen bezüglich der Datensicherheit oder Möglichkeiten zur Datenspeicherung und Internetnutzung gegenüber. Gleichzeitig gibt es weder in allen Schulen (in Deutschland) Informatikunterricht, noch haben alle aktuellen Schülerinnen und Schüler, geschweige denn diejenigen, die bereits vor vielen Jahren ihre Schullaufbahn abgeschlossen haben, Informatikunterricht gehabt. Im Fokus dieses Beitrags stehen daher diejenigen Personen, die während ihrer Schullaufbahn oder später im Beruf, keinen oder nur sehr wenig Kontakt mit Informatik und digitalen Medien erfahren haben. Solche Personen gelten im Folgenden als technikferne Erwachsene. Bei Befragungen von Teilnehmenden an Tablet-Workshops bezüglich ihrer Motivation in dem Workshop mitzumachen, äusserte sich, dass insbesondere die neuen Möglichkeiten der Kommunikation mit entfernt lebenden Freunden und Verwandten und somit auch der Wunsch an einer aktiven Teilhabe an der digitalen Welt für viele technikferne Erwachsene eine gute Motivation, sich mit den für sie neuen Technologien auseinanderzusetzen, bietet. Beispielsweise in Kursen an Volkshochschulen, könnten technikferne Erwachsene die nötigen Kompetenzen erwerben. Dabei stellt sich jedoch die Frage, um welche Kompetenzen es sich hierbei eigentlich handelt. Was sollten technikferne Erwachsene über Informatik wissen, um selbstständig an der durch Medien geprägten Welt teilhaben zu können?

Dieser Beitrag behandelt in Kapitel 2 das Vorgehen, welches angewendet wurde um die Informatik-Kompetenzen für technikferne Erwachsene zu ermitteln. In Kapitel 3 werden ausgewählte nationale und internationale Schulcurricula, Rahmenbedingungen und Empfehlungen sowie deren Tragweite in den entsprechenden Ländern, sowie aktuelle Trends und Themen in Deutschland betrachtet. Kapitel 4 beschäftigt 
sich mit den Interessen von technikfernen Erwachsenen, welche mit Hilfe eines Fragebogens ermittelt wurden. In Kapitel 5 werden die Kompetenzen vorgestellt, welche technikferne Erwachsene erwerben sollten. Kapitel 6 fasst letztlich zusammen und gibt einen Ausblick zur möglichen Nutzung der zuvor gewonnenen Erkenntnisse.

\section{Methode und Vorgehen}

Zur Ermittlung derjenigen Informatik-Kompetenzen, die technikferne Erwachsene, hier im Folgenden insbesondere Personen ab 50 Jahren, erwerben sollten wurde ein mehrstufiges Verfahren angewendet. In einem ersten Schritt wurden nationale und internationale Standards für Informatik in der Schule sowie aktuelle Themen der Informatik, die in Gesellschaft und Politik in Deutschland eine aktuelle Rolle spielen gesammelt. Da einige Kompetenzen in verschiedenen Standards vertreten sind, wurden diese zusammengefasst. Weiterhin wurden solche Kompetenzen, die gleich sind, jedoch in unterschiedlich Stufen vertreten sind, ebenfalls zusammengefasst und in der Stufe, in der sie zum ersten mal auftreten, eingeordnet. Ausgenommen werden für die weitere Betrachtung Kompetenzen mit Programmierbezug. Programmierung ist kein Bestandteil der KMK Strategie, die die Pflichtkompetenzen für die Bildung in der digitalen Welt behandelt und welche alle Schülerinnen und Schüler, welche ab dem Schuljahr 2018/2019 eingeschult werden, innerhalb ihrer Schullaufbahn erwerben sollen (Sekretariat der Kultusministerkonferenz 2017).

Basierend auf den Themengebieten und Kompetenzen dieser nationalen und internationalen Standards wurde in einem zweiten Schritt ein Fragebogen mit 52 Items zum Interesse der Teilnehmenden im Bezug auf die jeweiligen Themengebiete, die in den Kompetenzen angesprochen werden, erstellt. An dieser Fragebogenstudie nahmen 123 Personen ab 50 Jahren teil. Mithilfe dieses Fragebogens konnte ein Eindruck über die Interessensgebiete der Zielgruppe gewonnen werden. Das Interesse wurde dabei mit Hilfe einer 6-stufigen Likert-Skala ermittelt, wobei 6 ein sehr hohes Interesse bedeutet. Um einen zusätzlichen Indikator für schwer verständliche Fragen zu haben, wurde zusätzlich ein Fragezeichen-Feld eingeführt. Hierdurch sollte vermieden werden, das Teilnehmende, die eine Frage nicht vollständig verstehen, diese mit geringem Interesse bewerten und so die Endergebnisse verfälschen.

In einem dritten Schritt wurden die Ergebnisse aus der Analyse bestehender Standards, aktueller Themen und der Fragebogenstudie in Inforamtikkompetenzen für technikferne Erwachsene überführt. Die Basis für dieses Set an Kompetenzen bilden diejenigen Kompetenzen, welche in Standards von mindestens zwei unterschiedlichen Ländern vertreten waren. Aufbauend auf diese so entstandene Basis wurden diejenigen Kompetenzen hinzugefügt, an denen die Zielgruppe ein erhöhtes Interesse gezeigt hat. Dafür wurden Kompetenzen hinzugefügt, welche Themen behandeln, bei denen das Interesse bei der Umfrage sowohl im Mittelwert als auch im Median bei 
mindestens 4 lag. Weiterhin wurden Kompetenzen mit signifikant höherem Interesse bei Nicht-Tabletnutzenden gegenüber Tabletnutzenden aufgenommen. Aufgrund der Aktualität des Themas Urheberrecht wurden alle nicht programmierbezogenen Kompetenzen, aus dem Unterbereich Offene Materialien und Urheberrecht ebenfalls aufgenommen. Am Ende dieses Prozesses lagen Kompetenzen für vier von fünf Bereichen vor. Da der Bereich Sprachen und Automaten fast ausschliesslich in deutschen Richtlinien zu finden ist und auch bei der Zielgruppe kein erhöhtes Interesse besteht, ist keine aussagekräftige Grundlage für die Auswahl vorhanden. Es wurden letztlich die Kompetenzen aus der untersten Stufe (K-2), sowie eine Kompetenz, die sowohl in den Bildungsstandards der GI als auch in den Vorgaben der KMK vertreten sind, aufgenommen.

\section{Informatik in der Schule und aktuelle Themen in Deutschland}

\section{Informatik in der Schule}

Um eine möglichst umfassende Übersicht im Hinblick auf Informatik-Kompetenzen, die in der Schule erlangt werden sollten zu erhalten, werden nationale und internationale Schulcurricula, Standards und Empfehlungen betrachtet. Als nationale Dokumente aus Deutschland werden die Bildungsstandards der Gesellschaft für Informatik (Best et al. 2017; GI Gesellschaft für Informatik 2008; Röhner et al. 2016), Die Einheitliche Prüfungsanforderung Informatik der Kultusministerkonferenz (Ständige Kultuministerkonferenz 2004) sowie die Strategie «Bildung in der digitalen Welt» der Kultusministerkonferenz (Sekretariat der Kultusministerkonferenz 2017) vorgestellt. Im internationalen Bereich, werden Schulcurricula, Standards und Empfehlungen aus der Schweiz, den USA und England betrachtet. Dabei wurden diese Dokumente basierend auf ihrer Verfügbarkeit ausgewählt, insbesondere wurden Dokumente aus dem deutsch- und englischsprachigen Raum gewählt, um zu gewährleisten, dass es nicht zu sprachlichen Fehlinterpretationen kommt.

\section{Nationale Standards}

Aus nationaler Sicht wurden hier insbesondere die Bildungsstandards der Gesellschaft für Informatik (GI) herangezogen. Diese Standards sind sowohl für den Primarbereich (Best etal. 2017) als auch für den Sekundarbereich I und II (GI Gesellschaft für Informatik 2008; Röhner et al. 2016) verfügbar und bieten so einen einheitlichen Überblick für Informatik-Kompetenzen in allen Schulstufen in Deutschland. Innerhalb dieser Standards werden die einzelnen Kompetenzen in fünf Inhaltsbereiche und fünf Prozessbereiche eingeteilt, welche ineinandergreifen. Bei den Inhaltsbereichen 
handelt es sich um Information und Daten, Algorithmen, Sprachen und Automaten, Informatiksysteme sowie Informatik, Mensch und Gesellschaft. Die Prozessbereiche gliedern sich in Modellieren und Implementieren, Begründen und Bewerten, Strukturieren und Vernetzen, Kommunizieren und Kooperieren sowie Darstellen und Interpretieren. Bei gemeinsamer Betrachtung der drei vorhandenen Standards (Primarbereich, Sekundarstufe I und Sekundarstufe II), entsteht ein Gesamtüberblick über Kompetenzen, über welche Schülerinnen und Schüler nach der Jahrgangsstufe 2, 4, 7, 10 und 12 verfügen sollten. Die Standards richten sich hierbei an «Informatiklehrerinnen und -lehrer, an Entscheidungstragende in der Bildungsadministration und an diejenigen, die Informatiklehrkräfte in Universitäten oder Studienseminaren ausbilden» (GI Gesellschaft für Informatik 2008, V; vgl. Best et al. 2017; vgl. Röhner et al. 2016)

Ebenfalls betrachtet wurde die Einheitliche Prüfungsordnung in der Abiturprüfung (EPA) für das Fach Informatik, welche im Jahr 2004 durch die Kultusministerkonferenz beschlossen wurde. Dieses Dokument befasst sich insbesondere mit fachlichen und methodischen Kompetenzen, die bis zum Schulabschluss erworben werden sollen, bzw. als Kompetenzen für die abschliessende Prüfung im Fach Informatik vorausgesetzt werden. (Ständige Kultusministerkonferenz 2004)

Im Jahr 2016 verabschiedete die Kultusministerkonferenz zudem eine Strategie unter dem Motto «Bildung in der digitalen Welt» (Sekretariat der Kultusministerkonferenz 2017). In dieser Strategie werden Kompetenzen in den sechs Kompetenzbereichen Suchen, Verarbeiten und Aufbewahren, Kommunizieren und Kooperieren, Produzieren und Präsentieren, Schützen und sicher Agieren, Problemlösen und Handeln sowie Analysieren und Reflektieren beschrieben, die in der zunehmend digitalisierten Welt immer stärker von Bedeutung werden. Die dort enthaltenen Kompetenzen sollen allen Schülerinnen und Schülern in Deutschland, die ab dem Schuljahr 2018/2019 eingeschult werden, innerhalb der Pflichtschulzeit vermittelt werden (Sekretariat der Kultusministerkonferenz 2017).

Während die Bildungsstandards der GI als Empfehlungen zu verstehen sind, an denen sich Schulen im Optimalfall orientieren sollen, bieten die EPA und die Strategie zur Bildung in der digitalen Welt einheitliche Vorgaben für den (Informatik-) Unterricht in ganz Deutschland.

\section{Internationale Standards}

Auf internationaler Ebene wurden die K-12 Computer Science Standards aus den USA (Tucker 2003; Grover und Pea 2013; Seehorn et al. 2011; «Framework Statements by Grade Band» 0.J.), der Lehrplan 21 aus der Schweiz («Lehrplan 21» 0.J.), das National Curriculum aus England (UK DFE 2013; Berry 2013), sowie das Curriculum for Schools and Programme of Teacher Development der UNESCO mit Mitwirkenden aus 
Australien, den Niederlanden, der Schweiz, Belgien, USA, Grossbritannien, Singapur und Russland (Unesco 2002) betrachtet. Dabei wurden Dokumente aus unterschiedlichen Ländern gewählt, um zum einen möglichst viele Kompetenzen zu erhalten und zum anderen einen Eindruck davon zu erhalten, welche Kompetenzen in unterschiedlichen Ländern von Bedeutung sind. Neben den bereits erwähnten Kriterien der Verfügbarkeit und Sprache der Dokumente lag bei der Auswahl ein weiterer Fokus darauf Standards zu finden, die möglichst das gesamte Spektrum einer Schullaufbahn abdecken, damit diese im Anschluss differenzierter mit den nationalen Standards verglichen werden können.

Das K-12 Computer Science Curriculum der Computer Science Teachers Association (CSTA) beinhaltet Kompetenzen für die Bereiche Informatiksysteme, Netzwerk und Internet, Daten und Auswertung, Algorithmen und Programmierung sowie Auswirkungen von Informatik. Aufgeteilt ist das Curriculum, ähnlich wie die GI Standards in fünf Klassenstufen, K-2, 3-5, 6-8, 9-10 und 11-12. Damit entsprechen sie, weitestgehend den Stufen der GI Standards. Unterschiede in den Stufen sind auf die Unterschiede in den jeweiligen Schulsystemen in Deutschland und den USA zurückzuführen. Ebenfalls verbindet beide Standards die Intention ein Set an grundlegenden Informatik-Kompetenzen für alle Schülerinnen und Schüler bereitzustellen. (Tucker 2003; Grover und Pea 2013; Seehorn et al. 2011; «Framework Statements by Grade Band» o.J.)

Der Lehrplan 21 aus der Schweiz wurde zwischen 2010 und 2014 von der Deutschschweizer Erziehungsdirektoren-Konferenz erarbeitet und wird in den Lehrplänen der 21 deutsch- und mehrsprachigen Kantone der Schweiz eingesetzt. Er richtet sich an Schülerinnen und Schüler vom Kindergarten bis zur 9. Klasse. Dabei sind die Klassenstufen in drei Zyklen, K-2, 3-6, 7-9, unterteilt. Der Lehrplan 21 verfolgt zum einen das Ziel Schülerinnen und Schüler dazu zu befähigen, «Medien [zu] verstehen und verantwortungsvoll nutzen zu können» («Lehrplan 21» o.J.) sowie «Grundkonzepte der Informatik [zu] verstehen und zur Problemlösung ein[zu]setzen» («Lehrplan 21» o.J.). («Lehrplan 21» 0.J.)

Das National Curriculum für England ist in vier Klassenstufen unterteilt, so genannte Key Stages. Key Stage 1 umfasst hierbei die ersten beiden Schuljahre, Key Stage 2 die Schuljahre 3-6, Key Stage 3 die Schuljahre 7-9 und Key Stage 4 die Schuljahre 10 und 11. Das Curriculum wurde im Jahr 2013 veröffentlicht und gehört zum Pflichtprogramm für Schulen in England. Das Ziel der nicht weiter in Kategorien unterteilten Kompetenzen ist dabei, dass alle Schülerinnen und Schüler Grundlegende Prinzipien und Konzepte der Informatik verstehen und anwenden können. (UK DFE 2013; Berry 2013)

Das Curriculum for Schools and Programme of Teacher Development der UNESCO hat bereits 2002 das stetige Voranschreiten von Informations- und Kommunikationstechnologien zum Anlass genommen ein Curriculum für weiterführende Schulen zu 
entwickeln, um mit internationalen Trends Schritt zu halten. Die angegebenen Kompetenzen sind nicht weiter in Klassenstufen unterteilt, verteilen sich jedoch auf vier so genannte Module. Die Module behandeln die Themen ICT Literacy, Application on ICT in Subject Areas, Infusing ICT across the Curriculum sowie ICT Specialization. Das Hauptziel hinter diesem Curriculum ist «to ensure that all countries, both developed and developing, have access to the best educational facilities necessary to prepare young people to play full roles in modern society and to contribute to a knowledge nation» (Unesco 2002, 8). In dieser Formulierung wird die Bedeutung von Informatik-Kompetenzen für die Teilhabe an der modernen, digitalen Gesellschaft deutlich (Unesco 2002).

\section{Aktuelle Themen in Deutschland}

Um zusätzlich zu den Vorgaben für den Informatikunterricht auch die aktuellen Themen der digitalen Welt zu betrachten, wurden zusätzlich die Wahlprogramme der Parteien zur Bundestagswahl 2017 in Deutschland (2017) analysiert. Die zunehmende Digitalisierung in allen Lebensbereichen ist auch in diesen ein immer wiederkehrendes Thema. Zudem handelt es sich bei den, in den Wahlprogrammen auftretenden Themen, um Thematiken die häufig in den Nachrichten zu finden sind, daher ist anzunehmen, dass diese Themen auch technikfernen Erwachsenen nicht fremd sind. Bei der Analyse konnte insbesondere die Bedeutung der aktiven Teilhabe an der Gesellschaft in einer digitalisierten Welt wiedergefunden werden. Neben dem allgemeinen Einsatz und der Nutzung von Informatiksystemen, stehen konkret Themen wie Internet, Datenschutz und Datensicherheit sowie der kritische Umgang mit Informatiksystemen und Informationen aus dem Internet im Vordergrund. Es fällt auch auf, dass diese Hauptaspekte ebenfalls in sowohl nationalen als auch internationalen Standards für den Informatikunterricht wiederzufinden sind. Anders sieht dies bei sehr speziellen, ebenfalls vereinzelnd auftretenden Themen aus. Dabei handelt es sich um Kenntnisse über die Funktionsweise von Sensoren, Speichern, informationstechnischer Systeme sowie künstlicher Intelligenz. («Wahlprogramme Bundestagswahl 2017 - Bundestagswahl 2017» 2017)

Ebenso weitere aktuelle und wichtige Themen, die aktuell in der EU und somit auch in Deutschland sehr präsent sind, sind die neue Datenschutz Grundverordnung (DSGVO) («Datenschutz-Grundverordnung (EU-DSGVO) als übersichtliche Website» o.J.) und die EU-Urheberrechtsreform («EUR-Lex» O.J.). Auch diese Thematiken waren bereits in den Standards zu finden und sollten bei der Ermittlung der InformatikKompetenzen für technikferne Erwachsene nicht vernachlässigt werden. 


\section{Interessen von technikfernen Erwachsenen}

Es sei zu beachten, dass im Folgenden nur die Fragen, die auf erhöhtes Interesse gestossen sind diskutiert werden. Ein erhöhtes Interesse wurde zu Fragen aus den Themengebieten Datenschutz und Datensicherheit, Organisation von Daten und Dokumenten, Umgang mit Problemen, Kommunikation mit Informatiksystemen, Internet und Netzwerke sowie Informatiksysteme geäussert. Von erhöhtem Interesse wird hier gesprochen, wenn Mittelwert und Median der gegebenen Antworten für eine Frage bei mindestens 4 liegen. Im Bereich Datenschutz und Datensicherheit viel auf, dass das Interesse am Thema allgemein recht hoch ist, im Mittel wurden Werte zwischen 4,1 und 5,1 (Median bei 5 bzw. 6) erzielt. Allerdings sinkt das Interesse bezüglich Massnahmen und Mechanismen zu Datenschutz und Datensicherheit auf einen mittleren Wert von 3,0 und 3,1 (Median bei 3 bzw. 4). Im Themenbereich Organisation von Daten und Dokumenten wurde das Interesse mit Mittelwerten zwischen 4,1 und 4,8 (Median bei 5 bzw. 6) geäussert. Dabei stehen Aspekte wie abspeichern und wiederfinden von Daten, Schutz vor versehentlichem Löschen von Dateien sowie unterschiedliche Möglichkeiten zur Datenspeicherung im Vordergrund. Im Themenbereich Umgang mit Problemen, in welchem die Interpretation von Fehlermeldungen in der Nutzung von Informatiksystemen im Vordergrund steht, wurde ein Mittelwert von 4,5 und ein Median von 5 erreicht. In den Themenbereichen Kommunikation mit Informatiksystemen und Kommunikation über Informatiksysteme wurden insbesondere hohe Werte erreicht, wenn es darum ging, Informatiksysteme zu nutzen, um so mit anderen in Kontakt zu treten und zu bleiben. Auch das mitreden wollen, war ein wichtiger Aspekt. Hier wurden Mittelwerte zwischen 4,0 und 4,5 und ein Median von 4 bzw. 5 erreicht. Daraus wird deutlich, dass auch technikferne Erwachsene aktives Mitglied der digitalen Gesellschaft sein möchten. Im Themenbereich Internet und Netzwerke erzielten erneut Fragen in Richtung Datenschutz, z.B. richtiges An- und Abmelden, sichere Passwörter oder das Erkennen von Werbung eine wichtige Rolle. Ebenso wurde bezüglich dem Finden, Beschaffen und Bewerten von Informationen aus dem Internet ein erhöhtes Interesse gezeigt. Die Mittelwerte in diesem Themenbereich liegen zwischen 4,0 und 4,6, der Median bei 4 bzw. 5. Letztlich lösten im Themenbereich Informatiksysteme diejenigen Fragen, in denen es um die richtige Nutzung und Interaktion mit den Geräten geht das höchste Interesse aus. Dabei wurden für den Mittelwert Werte zwischen 4,0 und 4,6 und für den Median von 4 bzw. 5 erreicht.

Zusätzlich konnte festgestellt werden, dass das Interesse von Nicht-Tabeltnutzenden bei den folgenden Themen signifikant höher war, als bei den Tabletnutzenden. Bei den Themen handelt es sich um unterschiedliche Darstellungsformen für Daten und deren Interpretation, z.B. in Diagrammen und Statistiken, das Verständnis von Funktions- und Arbeitsweisen von Informatiksystemen sowie des Internets und der Bewertung der Qualität von Informationen aus dem Internet. 
Was technikferne Erwachsene über Informatik wissen sollten

Basierend auf dem in Kapitel 2 beschriebenen Prozess ergeben sich Kompetenzen in den fünf, an den Inhaltsbereichen der GI-Bildungsstandards orientierten, Bereichen. Es ist jeweils «Technikferne Nutzerinnen und Nutzer ...» an den Anfang zu setzen. Den folgenden Tabellen können die Unterthemen der Bereiche sowie die jeweiligen Kompetenzen entnommen werden. Zusätzlich wird für jede Kompetenz angegeben, in welchen der betrachteten Standards sie zu finden sind, sowie in welchen Schulstufen sie dort jeweils angesiedelt sind. Der Bereich Informationen und Daten ist in Tabelle 1 dargestellt, der Bereich Algorithmen in Tabelle 2, der Bereich Sprachen und Automaten in Tabelle 3, der Bereich Informatiksysteme in Tabelle 4 und der Bereich Informatik, Mensch und Gesellschaft in Tabelle 5. Alle Tabellen befinden sich im Anhang hinter dem Literaturverzeichnis.

\section{Zusammenfassung und Ausblick}

Nationale und internationale Schulcurricula bieten eine Basis für Kompetenzen, welche auch technikferne Erwachsene, also solche Personen, die keine informatische Bildung in der Schule, im Studium oder in der beruflichen Aus- und Weiterbildung erhalten haben, erwerben sollten. Hierzu wurden aus den Richtlinien für den Informatikunterricht in der Schule die Kompetenzen gewählt, die in mindestens zwei Ländern vertreten waren. Erweitert wurde die Basis durch Kompetenzen, die aktuell relevante Themengebiete, und Themengebiete mit erhöhtem Interesse bei der Zielgruppe wiederspiegeln. Auf diese Weise entstand ein Kompetenzset mit grundlegenden Informatik-Kompetenzen für technikferne Erwachsene. Durch diese werden neben der Bedienfähigkeit von Informatiksystemen auch informatische Konzepte abgedeckt. Hierdurch wird eine Übertragbarkeit der Kompetenzen von den digitalen Medien von heute auf die digitalen Medien von morgen, sowie eine aktive Teilhabe an der durch digitale Medien geprägten Welt ermöglicht.

Im Weiteren gilt es zu beforschen, auf welche Art und Weise die Themen in Form von Lernapplikationen aufbereitet werden müssen, damit sie für die Zielgruppe verständlich sind. Ein denkbares Szenario wäre hier die Entwicklung eines WorkshopKonzepts für beispielsweise Volkshochschulen oder die Entwicklung von eigenständigen, mobilen Lernapplikationen zum selbständigen Lernen. In beiden Szenarien ist ein Lernen direkt am Gerät sinnvoll, um das Erlernte direkt mit dem Gerät und an dem Gerät zu üben. 


\section{Literatur}

Berry, Miles. 2013. «Computing in the national curriculum. A guide for primary teachers». Computing at school.

Best, Alexander, Christian Borowski, Karin Büttner, Rita Freudenberg, Martin Fricke, Kathrin Haselmeier, Henry Herper, et al. 2017. «Kompetenzen für informatische Bildung im Primarbereich». Beilage zu LOG IN 38 (189/190). https://doi.org/10.13140/RG.2.2.22433.45922.

«Datenschutz-Grundverordnung (EU-DSGVO) als übersichtliche Website». o.J. DatenschutzGrundverordnung (DSGVO). Zugegriffen 11. Januar 2019. https://dsgvo-gesetz.de/.

«EUR-Lex». o.J. https://eur-lex.europa.eu/legal-content/DE/TXT/?uri=CELEX\%3A52016PC0593.

«Framework Statements by Grade Band». o.J. K12cs.Org. https://k12cs.org/framework-statements-by-grade-band/.

GI Gesellschaft für Informatik. 2008. «Grundsätze und Standards für die Informatik in der Schule. Bildungsstandards Informatik für die Sekundarstufe I». LOG IN 28 (150/151). https:// gi.de/fileadmin/Gl/Hauptseite/Aktuelles/Meldungen/2016/Bildungsstandards_2008.pdf.

Grover, Shuchi, und Roy Pea. 2013. «Computational thinking in K-12: A review of the state of the field». Educational Researcher 42 (1): 38-43. https://doi.org/10.3102/0013189X12463051.

«Lehrplan 21». o.J. https://v-ef.lehrplan.ch/.

Röhner, Gerhard, Torsten Brinda, Volker Denke, Lutz Hellmig, Theo Heußer, Arno Pasternak, Andreas Schwill, und Monika Seiffert. 2016. «Bildungsstandards Informatik für die Sekundarstufe II». Beilage zu LOG IN, Nr. 183/184. https://gi.de/fileadmin/GI/Hauptseite/Aktuelles/Meldungen/2016/Bildungsstandards-Informatik-Sekll.pdf.

Seehorn, Deborah, Stephen Carey, Brian Fuschetto, Irene Lee, Daniel Moix, Dianne O'GradyCunniff, Barbara Boucher Owens, Chris Stephenson, und Anita Verno. 2011. «CSTA K-12 Computer Science Standards: Revised 2011».

Sekretariat der Kultusministerkonferenz. 2017. «Bildung in der digitalen Welt: Strategie der Kultusministerkonferenz». Berlin: KMK. Zugriff 16. https://www.kmk.org/fileadmin/Dateien/pdf/PresseUndAktuelles/2017/Strategie_neu_2017_datum_1.pdf.

Ständige Kultusministerkonferenz. 2004. «Einheitliche Prüfungsanforderungen Informatik». http://www. kmk.org/fileadmin/veroeffentlichungen_beschluesse/1989/1989_12_01-EPAInformatik. pdf.

Tucker, Allen. 2003. «A Model Curriculum for K-12 Computer Science: Final Report of the ACM K-12 Task Force Curriculum Committee». https://dl.acm.org/citation.cfm?id=2593247. 


\begin{tabular}{|c|c|c|}
\hline \multicolumn{3}{|c|}{ Kodierung und Dekodierung } \\
\hline$\cdots$ & $\begin{array}{l}\text { können die Risiken unverschlüsselter Datenübermittlung } \\
\text { und -speicherung abschätzen. }\end{array}$ & «Lehrplan 21» O.J. (8-10) \\
\hline$\cdots$ & $\begin{array}{l}\text { kodieren Daten in eine binäre Darstellung und interpretieren } \\
\text { binär dargestellte Elemente als Daten. }\end{array}$ & $\begin{array}{l}\text { Best et al. 2017; Tucker } 2003 \\
\text { (K-2) }\end{array}$ \\
\hline$\ldots$ & $\begin{array}{l}\text { entwickeln Vereinbarungen, um Daten zu verschlüsseln und } \\
\text { entschlüsseln. }\end{array}$ & $\begin{array}{l}\text { Best et al. } 2017 \text { (3-4); «Lehr- } \\
\text { plan 21» O.J. (5-7) }\end{array}$ \\
\hline$\ldots$ & $\begin{array}{l}\text { verstehen, wie Zahlen, Zeichen und Bilder im Binärformat } \\
\text { dargestellt werden können. }\end{array}$ & $\begin{array}{l}\text { UK DFE 2013; Seehorn et al. } \\
\text { 2011; Tucker } 2003(8-10)\end{array}$ \\
\hline \multicolumn{3}{|c|}{ Daten Organisation und Strukturierung } \\
\hline$\cdots$ & $\begin{array}{l}\text { können Methoden zur Datenreplikation unterscheiden und } \\
\text { anwenden (Backup, Synchronisation, Versionierung). }\end{array}$ & «Lehrplan 21» 0.J. (8-10) \\
\hline$\ldots$ & $\begin{array}{l}\text { sind in der Lage identische Kopien von Daten anzufertigen } \\
\text { und diese an verschiedenen Orten zu speichern, um sie vor } \\
\text { Verlust zu schützen. }\end{array}$ & UK DFE 2013 (8-10) \\
\hline$\ldots$ & $\begin{array}{l}\text { beschreiben Strategien, um einem Datenverlust vorzubeu- } \\
\text { gen. }\end{array}$ & $\begin{array}{l}\text { GI Gesellschaft für Informa- } \\
\text { tik } 2008(8-10)\end{array}$ \\
\hline$\ldots$ & $\begin{array}{l}\text { erklären, wie Daten verloren gehen können und kennen die } \\
\text { wichtigsten Massnahmen, sich davor zu schützen. }\end{array}$ & «Lehrplan 21» 0.J. (5-7) \\
\hline$\cdots$ & $\begin{array}{l}\text { unterscheiden zwischen lokaler und globaler Datenspeiche- } \\
\text { rung. }\end{array}$ & Best et al. 2017 (3-4) \\
\hline$\ldots$ & speichern Daten und unterscheiden Arten der Speicher. & $\begin{array}{l}\text { GI Gesellschaft für Informa- } \\
\text { tik } 2008 \text { (5-7) }\end{array}$ \\
\hline$\ldots$ & speichern Daten und finden Daten wieder. & $\begin{array}{l}\text { «Lehrplan 21» o.J. (K-2); } \\
\text { Best et al. } 2017 \text { (3-4) }\end{array}$ \\
\hline$\ldots$ & $\begin{array}{l}\text { können Dokumente so ablegen, dass auch andere sie wie- } \\
\text { derfinden. }\end{array}$ & «Lehrplan 21» 0.J. (8-10) \\
\hline$\ldots$ & erläutern, dass Dokumente aus Daten bestehen. & Best et al. 2017 (K-2) \\
\hline$\ldots$ & $\begin{array}{l}\text { kennen und verwenden Baumstrukturen am Beispiel von } \\
\text { Verzeichnisbäumen. }\end{array}$ & $\begin{array}{l}\text { «Lehrplan 21» o.J.; GI Ge- } \\
\text { sellschaft für Informatik } \\
2008 \text { (5-7) }\end{array}$ \\
\hline \multicolumn{3}{|c|}{ Präsentation und Visualisierung } \\
\hline$\cdots$ & $\begin{array}{l}\text { identifizieren und beschreiben Muster in der Datenvisualisie- } \\
\text { rung, um Vorhersagen zu treffen. }\end{array}$ & $\begin{array}{l}\text { Seehorn et al. 2011, «Frame- } \\
\text { work Statements by Grade } \\
\text { Band» o.J. (K-2) }\end{array}$ \\
\hline$\ldots$ & $\begin{array}{l}\text { reflektieren Darstellungen und Darstellungsformen sowie } \\
\text { ihre Auswahl kritisch. }\end{array}$ & Röhner et al. 2016 (11-12) \\
\hline$\cdots$ & $\begin{array}{l}\text { sammeln und präsentieren dieselben Daten in verschiede- } \\
\text { nen visuellen Formaten. }\end{array}$ & $\begin{array}{l}\text { Seehorn et al. 2011; «Frame- } \\
\text { work Statements by Grade } \\
\text { Band» o.J. (K-2); «Lehrplan } \\
\text { 21» o.J. (5-7); GI Gesell- } \\
\text { schaft für Informatik } 2008 \\
(8-10)\end{array}$ \\
\hline
\end{tabular}

Tab. 1.: Informatik-Kompetenzen für Technikferne Erwachsene im Bereich Informationen und Daten. 


\begin{tabular}{|c|c|c|}
\hline \multicolumn{3}{|c|}{ Identifikation und Verständnis von Algorithmen } \\
\hline$\ldots$ & benennen Algorithmen in ihrer Lebenswelt. & $\begin{array}{l}\text { Best et al. 2017; Seehorn } \\
\text { et al. 2011; «Framework } \\
\text { Statements by Grade Band» } \\
\text { o.J. (K-2) }\end{array}$ \\
\hline$\ldots$ & $\begin{array}{l}\text { können formale Anleitungen erkennen und ihnen folgen, z.B. } \\
\text { Rezepte, Choreographien). }\end{array}$ & $\begin{array}{l}\text { «Lehrplan 21» 0.J.; Seehorn } \\
\text { et al. } 2011 \text { (K-2) }\end{array}$ \\
\hline$\ldots$ & $\begin{array}{l}\text { verstehen, was Algorithmen sind, wie sie als Programme auf } \\
\text { digitalen Geräten implementiert sind und dass Programme } \\
\text { ausgeführt werden, indem sie genaue und eindeutige Anwei- } \\
\text { sungen befolgen. }\end{array}$ & $\begin{array}{l}\text { Berry } 2013 \text { UK DFE 2013; } \\
\text { «Framework Statements by } \\
\text { Grade Band» O.J. (K-2) }\end{array}$ \\
\hline$\ldots$ & $\begin{array}{l}\text { benennen und formulieren Handlungsvorschriften aus dem } \\
\text { Alltag. }\end{array}$ & $\begin{array}{l}\text { GI Gesellschaft für Informa- } \\
\text { tik 2008; «Lehrplan 21» O.J. } \\
(5-7)\end{array}$ \\
\hline$\ldots$ & $\begin{array}{l}\text { lesen und verstehen Handlungsvorschriften für das Arbeiten } \\
\text { mit Informatiksystemen. }\end{array}$ & $\begin{array}{l}\text { GI Gesellschaft für Informa- } \\
\text { tik 2008; «Lehrplan 21» O.J. } \\
(5-7)\end{array}$ \\
\hline$\ldots$ & $\begin{array}{l}\text { interpretieren Handlungsvorschriften korrekt und führen sie } \\
\text { schrittweise aus. }\end{array}$ & $\begin{array}{l}\text { GI Gesellschaft für Informa- } \\
\text { tik 2008; Berry } 2013 \text { (5-7) }\end{array}$ \\
\hline \multicolumn{3}{|c|}{ Vergleich von Algorithmen } \\
\hline$\ldots$ & $\begin{array}{l}\text { vergleichen mehrere Algorithmen für die gleiche Aufgabe } \\
\text { und bestimmen, welcher am besten geeignet ist. }\end{array}$ & $\begin{array}{l}\text { Seehorn et al. 2011; «Frame- } \\
\text { work Statements by Grade } \\
\text { Band» o.J. (3-4); «Lehrplan } \\
\text { 21» o.J. (5-7); UK DFE } 2013 \\
(8-10)\end{array}$ \\
\hline
\end{tabular}

Tab. 2.: Informatik-Kompetenzen für Technikferne Erwachsene im Bereich Algorithmen.

\begin{tabular}{|l|l|l|}
\hline \multicolumn{3}{|c|}{ Funktionsweise von Automaten } \\
\hline$\ldots$ & $\begin{array}{l}\text { beschreiben, dass Automaten interne Zustände besitzen, die } \\
\text { von aussen nicht sichtbar sind. }\end{array}$ & Best et al. 2017 (K-2) \\
\hline$\ldots$ & $\begin{array}{l}\text { erläutern, dass ein Automat regelgesteuert seine Zustände } \\
\text { verändert. }\end{array}$ & Best et al. 2017 (K-2) \\
\hline \multicolumn{3}{|c|}{ Interaktion mit Automaten } \\
\hline$\ldots$ & beschreiben ihre Interaktion mit Automaten. & Best et al. 2017 (K-2) \\
\hline \multicolumn{3}{|c|}{ Formaten in der realen Welt } \\
\hline$\ldots$ & $\begin{array}{l}\text { beschreiben Automaten in ihrer Lebenswelt als selbstständi- } \\
\text { ge Maschinen. }\end{array}$ & Best et al. 2017 (K-2) \\
\hline \multicolumn{3}{|c|}{. } \\
\hline$\ldots$ & $\begin{array}{l}\text { unterscheiden die Begriffe «Syntax» und «Semantik» und } \\
\text { erläutern sie an Beispielen. }\end{array}$ & $\begin{array}{l}\text { GI Gesellschaft für Informa- } \\
\text { tik 2008 (8-10); Sekretariat } \\
\text { der Kultusministerkonfe- } \\
\text { renz 2017 (11-12) }\end{array}$ \\
\hline
\end{tabular}

Tab. 3.: Informatik-Kompetenzen für Technikferne Erwachsene im Bereich Sprachen und Automaten. 


\begin{tabular}{|c|c|c|}
\hline \multicolumn{3}{|c|}{ Eingabe-Verarbeitung-Ausgabe (EVA) } \\
\hline$\ldots$ & $\begin{array}{l}\text { verwenden Standard Ein- und Ausgabegeräte zum erfolgrei- } \\
\text { chen Betrieb von Computern und verwandten Technologien. }\end{array}$ & Tucker 2003 (K-2) \\
\hline$\ldots$ & $\begin{array}{l}\text { erläutern die informatisch grundlegende Sicht des EVA- } \\
\text { Prinzips. }\end{array}$ & Best et al. 2017 (K-2) \\
\hline$\ldots$ & $\begin{array}{l}\text { ordnen Bestandteile eines Informatiksystems der Eingabe, } \\
\text { der Verarbeitung und der Ausgabe zu. }\end{array}$ & $\begin{array}{l}\text { GI Gesellschaft für Informa- } \\
\text { tik } 2008 \text { (5-7) }\end{array}$ \\
\hline$\ldots$ & $\begin{array}{l}\text { sind vertraut im Umgang mit Tastaturen und anderen Ein- } \\
\text { und Ausgabegeräten. }\end{array}$ & Tucker 2003 (3-4) \\
\hline \multicolumn{3}{|c|}{ Interaktion mit Informatiksystemen } \\
\hline$\ldots$ & interagieren mit Informatiksystemen. & Best et al. 2017 (K-2) \\
\hline$\cdots$ & $\begin{array}{l}\text { können Geräte ein- und ausschalten, Programme starten, be- } \\
\text { dienen und beenden sowie einfache Funktionen nutzen. }\end{array}$ & $\begin{array}{l}\text { Seehorn et al. 2011; «Lehr- } \\
\text { plan 21» 0.J. (K-2) }\end{array}$ \\
\hline$\ldots$ & $\begin{array}{l}\text { können mit grundlegenden Elementen der Bedienoberfläche } \\
\text { umgehen (Fenster, Menu, mehrere geöffnete Programme). }\end{array}$ & «Lehrplan 21» o.J. (K-2) \\
\hline$\ldots$ & arbeiten mit grafischen Benutzungsoberflächen. & \\
\hline \multicolumn{3}{|c|}{ Funktionsweise von Informatiksystemen } \\
\hline .. & $\begin{array}{l}\text { beschreiben, dass Informatiksysteme von Menschen entwi- } \\
\text { ckelte Anweisungen abarbeiten. }\end{array}$ & Best et al. 2017 (K-2) \\
\hline$\ldots$ & $\begin{array}{l}\text { erschliessen sich selbstständig neue Anwendungen und } \\
\text { Informatiksysteme. }\end{array}$ & $\begin{array}{l}\text { GI Gesellschaft für Informa- } \\
\text { tik 2008; Unesco } 2002 \text { (8-10) }\end{array}$ \\
\hline$\cdots$ & $\begin{array}{l}\text { vergleichen Abstraktionsebenen und Interaktionen zwischen } \\
\text { Anwendungssoftware, Systemsoftware und Hardware- } \\
\text { Schichten. }\end{array}$ & $\begin{array}{l}\text { Seehorn et al. } 2011 \text { (8-10); } \\
\text { «Framework Statements by } \\
\text { Grade Band» O.J.; Röhner et } \\
\text { al. } 2016 \text { (11-12) }\end{array}$ \\
\hline \multicolumn{3}{|c|}{ Probleme mit Informatiksystemen } \\
\hline$\ldots$ & $\begin{array}{l}\text { interpretieren Fehlermeldungen bei der Arbeit mit Informa- } \\
\text { tiksystemen und nutzen sie produktiv. }\end{array}$ & $\begin{array}{l}\text { GI Gesellschaft für Informa- } \\
\text { tik } 2008(8-10)\end{array}$ \\
\hline \multicolumn{3}{|c|}{ Internet und Netzwerke } \\
\hline$\cdots$ & $\begin{array}{l}\text { wissen, dass Computernetzwerke verwendet werden kön- } \\
\text { nen, um Menschen mit anderen Menschen, Orten, Informati- } \\
\text { onen und Ideen zu verbinden. }\end{array}$ & $\begin{array}{l}\text { «Framework Statements by } \\
\text { Grade Band» O.J. (K-2) }\end{array}$ \\
\hline$\cdots$ & $\begin{array}{l}\text { wissen, dass das Internet es Menschen ermöglicht, sich mit } \\
\text { anderen weltweit über viele verschiedene Verbindungspunk- } \\
\text { te zu verbinden. }\end{array}$ & $\begin{array}{l}\text { «Framework Statements by } \\
\text { Grade Band» O.J. (K-2) }\end{array}$ \\
\hline$\cdots$ & $\begin{array}{l}\text { kennen verschiedene Möglichkeiten, um Informationen, die } \\
\text { über Netzwerke gesendet und empfangen werden, vor unbe- } \\
\text { fugtem Zugriff zu schützen. }\end{array}$ & $\begin{array}{l}\text { «Framework Statements by } \\
\text { Grade Band» O.J. (5-7) }\end{array}$ \\
\hline$\ldots$ & $\begin{array}{l}\text { können Informationen aus verschiedenen Quellen gezielt } \\
\text { beschaffen, auswählen und hinsichtlich Qualität und Nutzen } \\
\text { beurteilen. }\end{array}$ & «Lehrplan 21» o.J. (5-7) \\
\hline$\ldots$ & $\begin{array}{l}\text { verstehen die grundsätzliche Funktionsweise von Suchma- } \\
\text { schinen (Auswahl und Ranking von Suchergebnissen). }\end{array}$ & $\begin{array}{l}\text { «Lehrplan 21» O.J.; Berry } \\
\text { 2013; UK DFE } 2013 \text { (5-7) }\end{array}$ \\
\hline$\ldots$ & unterscheiden lokale und globale Netze. & $\begin{array}{l}\text { GI Gesellschaft für Informa- } \\
\text { tik } 2008 \text { (5-7) }\end{array}$ \\
\hline
\end{tabular}




\begin{tabular}{|c|c|c|}
\hline$\ldots$ & $\begin{array}{l}\text { verstehen Computernetzwerke einschliesslich des Internets } \\
\text { und verstehen, wie sie mehrere Dienste wie das World Wide } \\
\text { Web anbieten können. }\end{array}$ & $\begin{array}{l}\text { Berry 2013; UK DFE } 2013 \\
(5-7)\end{array}$ \\
\hline$\ldots$ & $\begin{array}{l}\text { analysieren die Kommunikation und die Datenhaltung in } \\
\text { vernetzen Systemen und beurteilen diese auch unter den } \\
\text { Gesichtspunkten des Datenschutzes und der Datensicher- } \\
\text { heit. }\end{array}$ & $\begin{array}{l}\text { Röhner et al. 2016; «Frame- } \\
\text { work Statements by Grade } \\
\text { Band» o.J. (11-12) }\end{array}$ \\
\hline \multicolumn{3}{|c|}{ Mobile Geräte und das Internet } \\
\hline$\ldots$ & $\begin{array}{l}\text { kennen verschiedene Möglichkeiten, ihre mobile Geräte mit } \\
\text { dem Internet zu verbinden und wenden diese an. }\end{array}$ & neu erstellt \\
\hline$\ldots$ & $\begin{array}{l}\text { wissen, wie und wann sie die Netzwerke (WLAN) anderer } \\
\text { Personen nutzen können, oder wann andere Personen ihre } \\
\text { Netzwerke (WLAN) nutzen können. }\end{array}$ & neu erstellt \\
\hline$\ldots$ & $\begin{array}{l}\text { wissen, wie man Geräte mit anderen Geräten oder Kompo- } \\
\text { nenten verbinden kann. }\end{array}$ & neu erstellt \\
\hline \multicolumn{3}{|c|}{ Komponenten von Informatiksystemen } \\
\hline$\cdots$ & $\begin{array}{l}\text { erweitern bestehende Informatiksysteme mit Soft- und } \\
\text { Hardware Komponenten. }\end{array}$ & $\begin{array}{l}\text { GI Gesellschaft für Informa- } \\
\text { tik } 2008(8-10)\end{array}$ \\
\hline$\ldots$ & unterscheiden Betriebssystem und Anwendersoftware. & $\begin{array}{l}\text { GI Gesellschaft für Informa- } \\
\text { tik 2008; «Lehrplan 21» 0.J. } \\
\text { (5-7) }\end{array}$ \\
\hline \multicolumn{3}{|c|}{ Tool- und Technologie-Auswahl } \\
\hline$\ldots$ & $\begin{array}{l}\text { wählen und nutzen geeignete Software für eine Vielzahl von } \\
\text { Aufgaben und erkennen, dass Benutzer unterschiedliche } \\
\text { Bedürfnisse und Präferenzen für die von ihnen verwendete } \\
\text { Technologie haben. }\end{array}$ & $\begin{array}{l}\text { «Lehrplan } 21 » 0 . J . ; \mathrm{GI} \mathrm{Ge}- \\
\text { sellschaft für Informatik } \\
2008 \text { (5-7) }\end{array}$ \\
\hline$\ldots$ & $\begin{array}{l}\text { stellen fest, welche Technologie nützlich ist, und wählen die } \\
\text { geeigneten Werkzeuge und Technologieressourcen aus, um } \\
\text { eine Vielzahl von Aufgaben und Problemen zu lösen. }\end{array}$ & Tucker 2003 (3-4) \\
\hline$\ldots$ & $\begin{array}{l}\text { wählen geeignete Werkzeuge und Technologieressourcen } \\
\text { aus, um eine Vielzahl von Aufgaben zu erfüllen und Proble- } \\
\text { me zu lösen. }\end{array}$ & $\begin{array}{l}\text { Tucker 2003; GI Gesellschaft } \\
\text { für Informatik } 2008 \text { (5-7) }\end{array}$ \\
\hline
\end{tabular}

Tab. 4.: Informatik-Kompetenzen für Technikferne Erwachsene im Bereich Informatiksysteme.

\begin{tabular}{|l|l|l|}
\hline \multicolumn{2}{|c|}{ Informatik und Gesellschaft } \\
\hline$\ldots$ & $\begin{array}{l}\text { lernen die potenziellen Gefahren bei der Nutzung digitaler } \\
\text { Medien an Beispielen kennen. }\end{array}$ & $\begin{array}{l}\text { «Lehrplan 21» 0.J.; GI Ge- } \\
\text { sellschaft für Informatik } \\
2008 \text { (5-7) }\end{array}$ \\
\hline$\ldots$ & $\begin{array}{l}\text { bewerten Veränderungen (Vor- und Nachteile) in Alltags- und } \\
\text { Lebenswelt durch die Digitalisierung. }\end{array}$ & $\begin{array}{l}\text { Best et al. 2017; Seehorn et } \\
\text { al. 2011; «Framework State- } \\
\text { ments by Grade Band» o.J.); } \\
\text { Tucker 2003 (3-4) }\end{array}$ \\
\hline$\ldots$ & $\begin{array}{l}\text { beschreiben Chancen, Risiken und Missbrauchsmöglichkei- } \\
\text { ten von Informatiksystemen. }\end{array}$ & $\begin{array}{l}\text { Röhner et al. 2016; Ständige } \\
\text { Kultusministerkonferenz } \\
\text { 2004 (11-12) }\end{array}$ \\
\hline
\end{tabular}




\begin{tabular}{|c|c|c|}
\hline & wenden gesellschaftliche und ethische Vereinbarungen an. & $\begin{array}{l}\text { Best et al. 2017; Tucker } \\
\text { 2003; «Framework State- } \\
\text { ments by Grade Band» O.J. } \\
\text { (K-2) }\end{array}$ \\
\hline$\ldots$ & $\begin{array}{l}\text { erkennen akzeptables/unakzeptables Verhalten und kennen } \\
\text { eine Reihe von Möglichkeiten Bedenken hinsichtlich des } \\
\text { Inhalts und der Kontaktaufnahme zu melden. }\end{array}$ & $\begin{array}{l}\text { Tucker 2003; UK DFE 2013; } \\
\text { Berry } 2013 \text { (5-7) }\end{array}$ \\
\hline$\cdots$ & $\begin{array}{l}\text { zeigen Kenntnisse über die aktuellen Veränderungen der } \\
\text { Informationstechnologien und die Auswirkungen dieser } \\
\text { Veränderungen auf das tägliche Leben, den Arbeitsplatz und } \\
\text { die Gesellschaft. }\end{array}$ & $\begin{array}{l}\text { Seehorn et al. 2011; «Frame- } \\
\text { work Statements by Grade } \\
\text { Band» o.J.; Tucker } 2003 \\
\text { (5-7); GI Gesellschaft für } \\
\text { Informatik } 2008 \text { (8-10) }\end{array}$ \\
\hline \multicolumn{3}{|c|}{ Datensicherheit } \\
\hline$\ldots$ & $\begin{array}{l}\text { wenden Massnahmen an, um Daten vor ungewünschtem } \\
\text { Zugriff zu schützen. }\end{array}$ & Best et al. 2017 (K-2) \\
\hline$\ldots$ & $\begin{array}{l}\text { erklären, was Passwörter sind und warum wir sie verwen- } \\
\text { den. }\end{array}$ & Seehorn et al. 2011 (K-2) \\
\hline$\cdots$ & $\begin{array}{l}\text { verwenden sichere Passwörter, um Geräte und Informatio- } \\
\text { nen vor unbefugtem Zugriff zu schützen. }\end{array}$ & $\begin{array}{l}\text { Seehorn et al. 2011; «Frame- } \\
\text { work Statements by Grade } \\
\text { Band» o.J.) (K-2) }\end{array}$ \\
\hline \multicolumn{3}{|c|}{ Daten- und Informationsschutz } \\
\hline$\ldots$ & $\begin{array}{l}\text { nutzen die Technologie sicher und respektvoll und behan- } \\
\text { deln personenbezogene Daten vertraulich. }\end{array}$ & $\begin{array}{l}\text { «Framework Statements by } \\
\text { Grade Band» o.J.; UK DFE } \\
2013 \text { Berry } 2013 \text { (K-2) }\end{array}$ \\
\hline \multicolumn{3}{|c|}{ Kommunikation über Informatiksystemen } \\
\hline$\ldots$ & $\begin{array}{l}\text { kommunizieren über informatische Gegenstände und Bezie- } \\
\text { hungen in der Umgangssprache und zunehmend auch in der } \\
\text { fachgebundenen Sprache mit fachspezifischen Begriffen. }\end{array}$ & Best et al. 2017 (3-4) \\
\hline .. & $\begin{array}{l}\text { stellen Fragen und äussern sich begründet über informati- } \\
\text { sche Zusammenhänge unterschiedlicher Komplexität. }\end{array}$ & Best et al. 2017 (3-4) \\
\hline \multicolumn{3}{|c|}{ Kommunikation mit Informatiksystemen } \\
\hline$\ldots$ & nutzen E-Mail und Chat zum Austausch von Informationen. & $\begin{array}{l}\text { GI Gesellschaft für Informa- } \\
\text { tik } 2008 \text { (5-7) }\end{array}$ \\
\hline$\ldots$ & $\begin{array}{l}\text { verwenden elektronische Plattformen zum Austausch ge- } \\
\text { meinsamer Dokumente. }\end{array}$ & $\begin{array}{l}\text { GI Gesellschaft für Informa- } \\
\text { tik } 2008(5-7)\end{array}$ \\
\hline$\ldots$ & $\begin{array}{l}\text { kennen positive und negative Aspekte der Art und Weise, wie } \\
\text { Menschen mit Hilfe von Technologien kommunizieren. }\end{array}$ & $\begin{array}{l}\text { «Framework Statements by } \\
\text { Grade Band» o.J. (K-2) }\end{array}$ \\
\hline$\ldots$ & $\begin{array}{l}\text { nutzen synchrone und asynchrone Kommunikationsmög- } \\
\text { lichkeiten zum Austausch von Information und zu kooperati- } \\
\text { ver Arbeit. }\end{array}$ & $\begin{array}{l}\text { GI Gesellschaft für Informa- } \\
\text { tik } 2008 \text { (8-10) }\end{array}$ \\
\hline$\cdots$ & $\begin{array}{l}\text { beachten Umgangsformen bei elektronischer Kommunikati- } \\
\text { on und achten auf die Persönlichkeitsrechte anderer. }\end{array}$ & $\begin{array}{l}\text { GI Gesellschaft für Informa- } \\
\text { tik } 2008 \text { (5-7) }\end{array}$ \\
\hline
\end{tabular}




\begin{tabular}{|l|l|l|}
\hline \multicolumn{3}{|c|}{ Kooperation und Kollaboration } \\
\hline$\ldots$ & $\begin{array}{l}\text { arbeiten kooperativ und kollaborativ mit anderen zusam- } \\
\text { men, wenn sie Technologie einsetzen. }\end{array}$ & $\begin{array}{l}\text { Tucker 2003 (K-2); GI Gesell- } \\
\text { schaft für Informatik 2008 } \\
\text { (5-7) }\end{array}$ \\
\hline$\ldots$ & $\begin{array}{l}\text { kooperieren zur bzw. bei der Bearbeitung informatischer } \\
\text { Probleme. }\end{array}$ & $\begin{array}{l}\text { Best et al. 2017; Tucker 2003 } \\
\text { (3-4) }\end{array}$ \\
\hline \multicolumn{3}{|c|}{ Offene Materialien und Urheberrecht } \\
\hline$\ldots$ & $\begin{array}{l}\text { geben Attributionen, wenn sie die Ideen und Schöpfungen } \\
\text { anderer bei der Entwicklung von Programmen nutzen. }\end{array}$ & Seehorn et al. 2011 (K-2) \\
\hline$\ldots$ & $\begin{array}{l}\text { verwenden gemeinfreie oder unter Creative Commons ste- } \\
\text { hende Medien und kopieren oder verwenden kein Material, } \\
\text { das von anderen ohne Erlaubnis erstellt wurde. }\end{array}$ & $\begin{array}{l}\text { Seehorn et al. 2011; «Frame- } \\
\text { work Statements by Grade } \\
\text { Band» 0.J. (3-4) }\end{array}$ \\
\hline$\ldots$ & $\begin{array}{l}\text { respektieren die Eigentumsrechte an digitalen Werken. } \\
\text { Gl Gesellschaft für Informa- } \\
\text { tik 2008 (5-7) }\end{array}$ \\
\hline$\ldots$ & $\begin{array}{l}\text { kennen und beachten grundlegende Aspekte des Urheber- } \\
\text { rechts. }\end{array}$ & $\begin{array}{l}\text { Seehorn et al. 2011; Tucker } \\
\text { 2003; GI Gesellschaft für } \\
\text { Informatik 2008 (8-10) }\end{array}$ \\
\hline$\ldots$ & $\begin{array}{l}\text { diskutieren und bewerten wesentliche Aspekte des Daten- } \\
\text { schutz- und Urheberrechts anhand von Anwendungsfällen. }\end{array}$ & Röhner et al. 2016 (11-12) \\
\hline & \multicolumn{2}{|c|}{ Informatik und Mensch } \\
\hline$\ldots$ & $\begin{array}{l}\text { können Werbung erkennen und über die Zielsetzung der } \\
\text { Werbebotschaften sprechen. }\end{array}$ & «Lehrplan 21» 0.J. (K-2) \\
\hline
\end{tabular}

Tab. 5.: Informatik-Kompetenzen für Technikferne Erwachsene im Bereich Informatik, Mensch und Gesellschaft. 